\title{
LA RED TRANSEUROPEA DE TRANSPORTE Y EL (DES)EQUILIBRIO TERRITORIAL DE EUROPA. ENSEÑANZAS DE CARA A LA AMPLIACIÓN
}

\author{
David RAMOS PÉREZ \\ Universidad de Salamanca
}

\section{INTRODUCCIÓN.}

El programa de Red Transeuropea de Transporte (RTE-T) constituye el intento contemporáneo más ambicioso de dotar al continente europeo de una serie de corredores multimodales de transporte, superando así la tradicional planificación de infraestructuras desde una óptica nacional. En cierta medida, y con la perspectiva del tiempo histórico transcurrido, la RTE-T podría considerarse heredera de esa gran empresa del Imperio Romano que fue la construcción de una red de calzadas con una longitud cercana a los 85.000 kilómetros.

Sin embargo, no podemos olvidar que la RTE-T es sobre todo una respuesta estratégica a los cambios de la estructura económica que han afectado al transporte de pasajeros y mercancías durante la segunda mitad del siglo XX. El desarrollo de una economía de servicios, la dispersión de las actividades económicas, el aumento de la motorización y la segregación espacial de los usos del suelo, han incrementado la necesidad de los desplazamientos y las distancias recorridas. El proceso de integración económica de la UE no ha hecho sino acentuar estos patrones globales en el ámbito comunitario, potenciando el transporte internacional a larga distancia y las relaciones entre los principales nodos del sistema urbano.

Para la disciplina geográfica, el estudio de la RTE-T cobra especial relevancia desde la perspectiva de su impacto espacial, de su capacidad para profundizar en una mejor articulación del territorio europeo que ayude a atenuar los desequilibrios actualmente existentes. No en vano, una red de transporte termina siendo una organización espacial de un sistema de transporte, cuyo diseño, desarrollo y gestión no depende tanto de la demanda, de la capacidad tecnológica o de los recursos económicos disponibles, sino sobre todo del proyecto social e institucional predominante. Y la RTE-T es esencialmente un producto del proyecto comunitario.

En este contexto, el artículo presenta en primer lugar el proceso de gestación de la RTE-T en el ámbito comunitario y las justificaciones dadas para su puesta 
en marcha, planteando también su extensión al resto del continente por medio de la red TINA. Posteriormente, y después de un cuestionamiento teórico previo del binomio transporte-desarrollo, se procede a discutir la validez de los objetivos planteados en las orientaciones del programa, tomando como referencia varios estudios sobre el impacto territorial de la RTE-T así como el destino principal de la inversión en infraestructura de transporte. Los resultados de este análisis aportan una sólida base que permite evaluar algunos de los potenciales efectos derivados de la implantación de la red TINA en los países candidatos, especialmente aquellos relacionados con el equilibrio territorial. Paralelamente al desarrollo de la exposición se desgranan una serie de propuestas, que deberían ayudar a reorientar las actuaciones en materia de redes de transporte, sobre todo si realmente se concibe la RTE-T como una herramienta para avanzar en el refuerzo de la cohesión económica y social de Europa.

\section{LA IDEA DE RED TRANSEUROPEA DE TRANSPORTE. \\ 1. Origen y fundamentos de la Red Transeuropea de Transporte.}

La declaración sobre una Red Europea de Carreteras, firmada en Ginebra en 1950 por treinta y tres países bajo el auspicio de la Comisión Económica para Europa de las Naciones Unidas (UNECE), puede considerarse el primer referente de la actual Red Transeuropea de Transporte (RTE-T). De ese acuerdo surgió el compromiso de los distintos estados de prestar una especial atención a determinadas carreteras, los itinerarios $E$, que en conjunto constituían corredores de larga distancia conectando los extremos del continente. Sin embargo, hasta fechas relativamente recientes, la política comunitaria fue bastante limitada en cuanto a la planificación y desarrollo de redes de transporte desde una perspectiva supranacional, aún cuando el Tratado de Roma ya preveía la existencia de una Política Común de Transportes (PCT) ${ }^{1}$. En este sentido, desde 1957 hasta finales de los ochenta sólo cabe destacar tres actuaciones relevantes: en 1975 se comenzó a invertir en la infraestructura de las regiones menos desarrolladas, como consecuencia de la puesta en marcha del Fondo Europeo de Desarrollo Regional (FEDER); en 1978 se creó un Comité de Infraestructuras de Transporte, que prestó especial atención a ciertos estrangulamientos de las redes existentes; y a partir de 1982, por iniciativa del Parlamento Europeo, se abrió una línea presupuestaria que posibilitó modestas inversiones en proyectos de interés comunitario (CCE, 1992, 80; Ross, 1998, 183).

\footnotetext{
${ }^{1}$ Así lo recogen los antiguos artículos del 74 al 84, aunque tal PCT no será una realidad hasta la adopción en 1992 del Libro Blanco sobre El curso futuro de la política común de transportes, cuyo objetivo principal es alcanzar a largo plazo la denominada movilidad sostenible.
} 
Habrá que esperar a la comunicación de la Comisión Hacia unas redes transeuropeas: objetivos y aplicaciones posibles, formulada en 1989, para que aparezca el primer documento comunitario relativo a la posible adopción de un programa de redes transeuropeas de transporte, energía y telecomunicaciones. La Comisión justificó la necesidad de una política de redes transeuropeas basándose en cinco razones fundamentales, que con ciertas matizaciones y un mayor grado de refinamiento han pasado a formar parte del discurso oficial de las instituciones comunitarias: (i) el aumento previsible de los intercambios comunitarios como consecuencia del Mercado Único, (ii) la necesidad de interconectar las infraestructuras y servicios para que se adecuen a la nueva dimensión del mercado, (iii) la necesidad de tener en cuenta la dimensión comunitaria a la hora de concebir y desarrollar futuras redes, (iv) la necesidad de que la calidad de los servicios se corresponda con la magnitud del espacio europeo, (v) la necesidad de reducir las distancias entre todos los componentes del espacio comunitario (CCE, 1990, 6). Por tanto, la Comisión planteaba que las infraestructuras de transporte no estaban adaptadas a la nueva situación derivada de la entrada en vigor del Mercado Único, que potenciaba los desplazamientos internacionales intra-comunitarios, ya que dichas infraestructuras permanecían en gran medida segmentadas debido a que las competencias para su planificación y construcción recaían en los distintos Estados miembros.

No por casualidad estas afirmaciones son coincidentes con las preocupaciones manifestadas en los informes elaborados desde mediados de los ochenta por uno de los más potentes grupos de presión del continente, la European Round Table of Industrialists (ERT), representante de los intereses de empresas de la talla de Fiat, Bosch, Daimler Benz, Petrofina, Pirelli, Total y Volvo. De hecho, la propia Comisión sostenía que había evaluado la necesidad de las infraestructuras de transporte basándose entre otros, en los informes de "los circuitos económicos interesados, principalmente las organizaciones europeas más representativas de la industria" (CCE, 1990, 5). En las publicaciones Missing Links (1984) y Need for renewing transport infrastructure in Europe (1989), la ERT planteaba que las deficiencias que presentaban las redes de transporte podían reducir seriamente los beneficios económicos asociados a la puesta en marcha del Mercado Único, defendiendo un mayor gasto en infraestructuras y la necesidad de unas redes transeuropeas (RICHARDSON, 1997, 336 y ROSS, 1998, 49).

En este contexto, el Libro Blanco de 1992 sobre El curso futuro de la política común de transportes, permitió reforzar el concepto de red transeuropea de transporte en el ámbito comunitario, aprovechando el salto cualitativo que a partir de esa fecha experimentó la Política Común de Transportes. En esta nueva etapa, el desarrollo de las infraestructuras de transporte pasó a considerarse un elemento básico para alcanzar los objetivos de la PCT, especialmente aque- 
llos que hacen referencia al refuerzo y funcionamiento adecuado del mercado interno y al fortalecimiento de la cohesión económica y social (SCHMIDT Y GIORGI, 2001, 299). La revisión de los Tratados firmada en Maastricht en 1992 vino a confirmar el papel relevante asignado a las redes transeuropeas de transporte, telecomunicaciones y energía, al introducir un título XII (actualmente XV) en el Tratado de la Comunidad Europea, dedicado específicamente a las mismas. Este título obligaba a la elaboración de unas orientaciones en materia de redes transeuropeas, lo que en el caso de los transportes posibilitó la integración de los distintos esquemas modales que se habían desarrollado desde 1990.

Si bien el título XV vinculaba nuevamente las redes transeuropeas con los objetivos comunitarios del mercado interior y la cohesión económica y social, y hablaba de la necesidad de establecer enlaces entre las regiones insulares, enclavadas y periféricas y las regiones centrales, será el Libro Blanco sobre Crecimiento, competitividad y empleo de 1993 el que aporte nuevos razonamientos para justificar la necesidad de las redes, al concebirlas como un programa de tipo keynesiano de gran inversión en obra pública. En el corto plazo la construcción de la infraestructura generaría nuevos empleos, mientras a medio y largo plazo el incremento de la accesibilidad mejoraría la competitividad de la economía europea, con lo que se potenciaría su capacidad de creación de empleo (SICHELSCHMIDT, 1999: 172). De esta manera, se convertía a la RTE-T, junto con las redes de telecomunicaciones y energía, en herramienta clave para relanzar la economía de la UE, consumando una ya clásica relación entre transporte y desarrollo.

\section{Las orientaciones comunitarias de 1996 y la financiación de la RTE- T.}

Con estos precedentes, en 1996 se adoptaron finalmente las orientaciones comunitarias para el desarrollo de la red transeuropea de transporte (DOCE, 1996, 1-103), cumpliendo de esta manera el mandato del Tratado, aunque el proceso no estuvo exento de un serio enfrentamiento entre el Parlamento y el Consejo. El Parlamento, aprovechando sus nuevos poderes, criticó la forma poco democrática en la que se habían gestado las primeras etapas del proceso y la escasa atención que se prestaba a las cuestiones ambientales. Se pretendía así reorientar el programa de RTE-T, de tal manera que no fuera únicamente un instrumento al servicio del Mercado Único, sino sobre todo una herramienta dentro de una estrategia global de desarrollo sostenible, que permitiera alcanzar un mayor equilibrio entre ecología y economía (RICHARDSON, 1997, 338). Ello llevó a un proceso de conciliación, que permitió la entrada en vigor de las orientaciones, donde se recogieron algunas de las enmiendas propuestas por el Parlamento, como la necesidad de una evaluación estratégica de las repercusio- 
nes ambientales de la RTE-T, aunque no se incluyeron las cuotas de inversión por modos, que perseguían limitar el gasto en la red de carreteras a un máximo del $25 \%$ del total.

Las orientaciones comunitarias definen una RTE-T compuesta de infraestructuras de transporte, de sistemas de gestión de tráfico y de sistemas de localización y navegación. Las infraestructuras constituyen el núcleo fundamental del programa (MAPAS 1 y 2), comprendiendo $75.185 \mathrm{~km}$ de carreteras (el $23 \%$ de la red principal de la UE), $79.440 \mathrm{~km}$ de ferrocarriles (el 50\%), $20.000 \mathrm{~km}$ de vías navegables, 381 aeropuertos, 273 puertos marítimos y 210 puertos fluviales. Se identificaron también 14 proyectos prioritarios (MAPA 3) de los 35 propuestos por el grupo Christophersen en 1994, a los cuales los Consejos Europeos de Essen (1994) y Dublín (1996) atribuyeron especial importancia de cara a la consecución de los objetivos del programa.

Como resulta lógico, la decisión sobre las orientaciones recogía como objetivos la mayoría de los planteados en las comunicaciones previas, de tal manera que la RTE-T debería (artículo 2): (i) asegurar la movilidad sostenible de las personas y las mercancías, contribuyendo a reforzar la cohesión económica y social, (ii) ofrecer a los usuarios unas infraestructuras de calidad, (iii) incluir todos los modos de transporte, (iv) permitir la utilización óptima de las capacidades existentes, (v) favorecer la intermodalidad, (vi) ser económicamente viable, (vii) cubrir todo el territorio de la Comunidad, con especial énfasis en las regiones insulares, enclavadas y periféricas, y (viii) poder conectarse a las redes de terceros países.

Aunque no aparezca entre los objetivos, puede decirse que de forma implícita también se apostaba por un reequilibrio de las cuotas de mercado de los distintos modos, favoreciendo a aquellos generadores de un menor impacto ambiental, tal y como se insinuaba en el Libro Blanco de 1992 sobre la $\mathrm{PCT}^{2}$. Así, aunque los kilómetros de red viaria y ferroviaria incluidos en la RTE-T son similares, la inversión que se destina al ferrocarril prácticamente duplica la de la carretera, suponiendo el 50,5\% del gasto total previsto (CUADRO 1). Estas cifras son aún más dispares si sólo consideramos los proyectos prioritarios, donde se destina al tren de alta velocidad (TAV) el $80 \%$ del gasto previsto. Otra cuestión, como veremos más adelante, es si esta preferencia por el TAV es la más adecuada de cara a cumplir con los objetivos perseguidos.

\footnotetext{
${ }^{2}$ El Consejo Europeo de Gotemburgo de 2001 ya hizo una clara apuesta por conseguir una transferencia de pasajeros y mercancías de la carretera al ferrocarril, dentro de la estrategia europea de desarrollo sostenible (CCE, 2001a, 14). Esta recomendación fue totalmente integrada en el nuevo Libro Blanco sobre la PCT aparecido ese mismo año, en el que el reequilibrio de los modos de transporte es uno de sus pilares fundamentales (CCE, 2001b).
} 
Mapa 1. RTE-T en la UE ampliada: ferrocarril

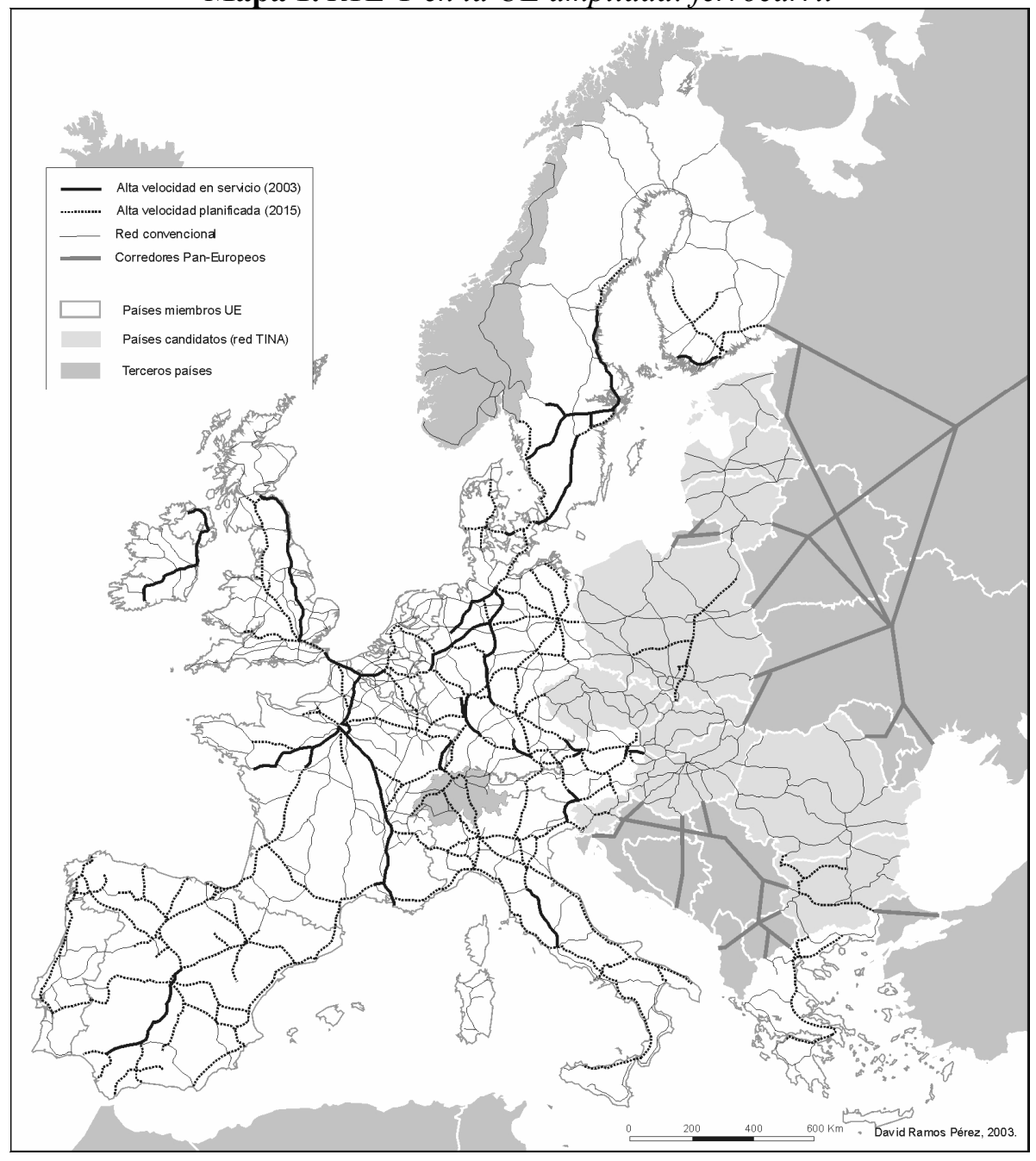

FUENTE: CCE, 2001d; TINA, 1999. Elaboración Propia. 
Mapa 2. RTE-T en la UE ampliada: carreteras

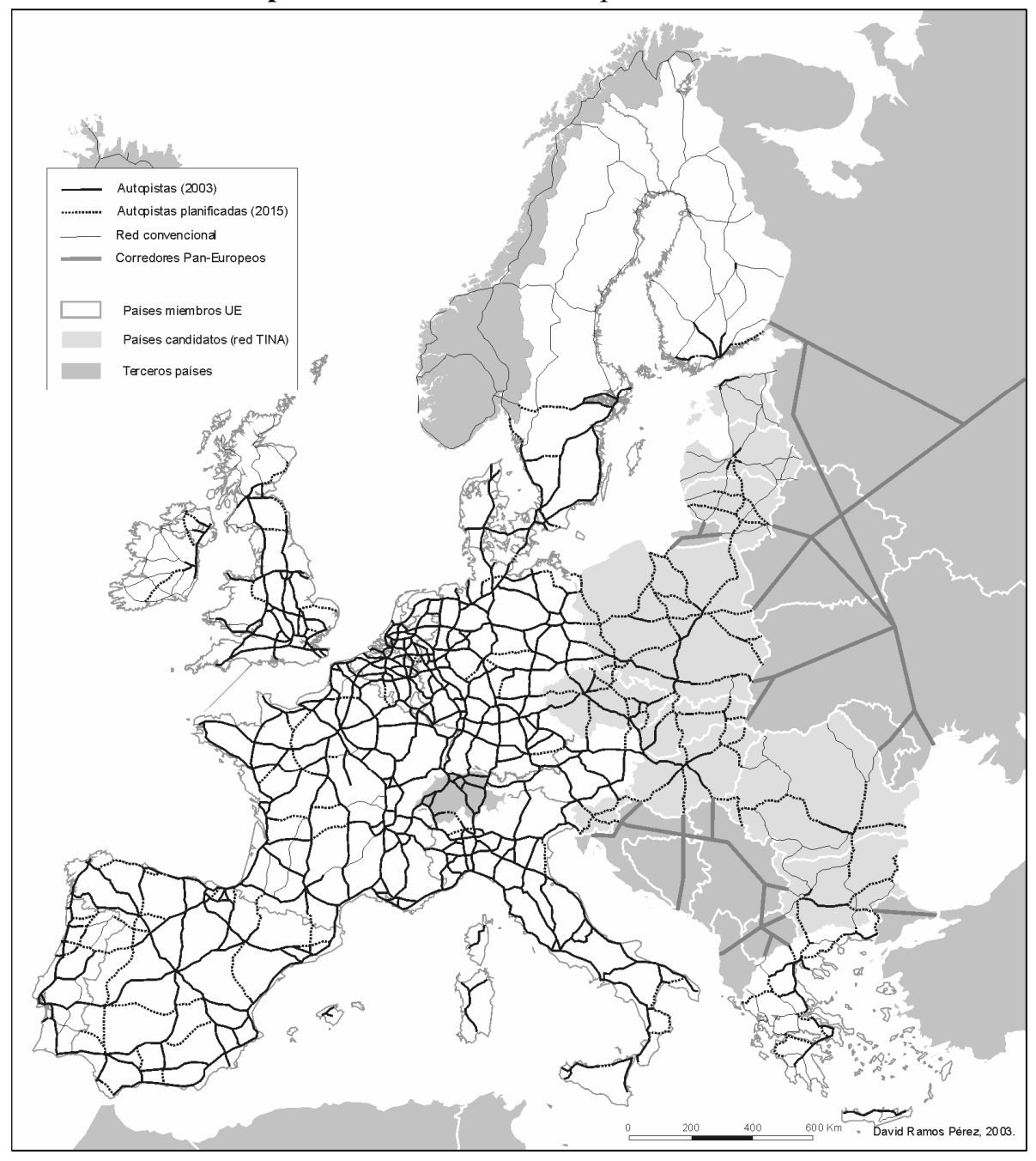

FUENTE: CCE, 2001d; TINA, 1999. Elaboración Propia. 
Cuadro 1. Composición y coste de la RTE-T (1996-2010)

\begin{tabular}{lrrr}
\hline \multicolumn{1}{c}{ Modos } & Longitud o $\mathrm{n}^{\mathrm{o}}$ & Coste $\left(^{*}\right)$ & $\%$ \\
\hline Red viaria & $75.185 \mathrm{~km}$ & $113.196,5$ & 26,0 \\
Red ferroviaria & $79.440 \mathrm{~km}$ & $219.749,3$ & 50,5 \\
Navegación interior & $20.000 \mathrm{~km}$ & $8.237,4$ & 1,9 \\
Aeropuertos & 381 & $56.214,6$ & 12,9 \\
Puertos marítimos & 273 & $38.159,5$ & 8,8 \\
Puertos fluviales & 210 & & 100,0 \\
\hline TOTAL & -- & $435.557,4$ & \\
\hline
\end{tabular}

(*) Estimaciones en millones de euros.

FUENTE: CCE, 2001, 4; PLANCO, 2003, 63.

Por otro lado, es evidente que la realización de un programa de la envergadura de la RTE-T depende de una elevada capacidad de financiación. No en vano, la Comisión maneja un presupuesto para el periodo 1996-2010 por encima de los 400.000 millones de euros, de los que más de cien mil corresponden a los 14 proyectos prioritarios. La mayoría de las inversiones son financiadas a partir de los presupuestos nacionales, que durante los años noventa han estado sujetos a fuertes restricciones de gasto, en buena parte debidas al proceso de convergencia impuesto para alcanzar la Unión Económica y Monetaria. De todos modos, la inversión en infraestructura de transporte se encuentra estabilizada desde principios de los ochenta, suponiendo como término medio el $1 \%$ del producto nacional bruto de los Estados miembros (CCE, 2001b, 147). No obstante, la aportación comunitaria ha sido cuantiosa, no tanto a través de la línea presupuestaria habilitada en 1995 para financiar proyectos de interés común (DOCE, 1995, 1-7), sino sobre todo por la contribución del FEDER y el Fondo de Cohesión (CUADRO 2). Las ayudas totales se aproximaron a los 14.000 millones de euros durante el periodo 1994-1999, lo que supone un 2,8\% del gasto comunitario, porcentaje que se reducirá ligeramente durante la etapa 2000-2006.

Cuadro 2. Financiación de la RTE-T hasta 2006 (*)

\begin{tabular}{lrr}
\hline \multicolumn{1}{c}{ Fondos } & $1994-1999$ & $2000-2006$ \\
\hline Presupuestos nacionales & 16.735 & 64.167 \\
\hline Presupuesto comunitario & 13.843 & 18.200 \\
Fondo RTE-T & 2.043 & 4.200 \\
Fondo Cohesión & $8.300^{\mathrm{b}}$ & 9.000 \\
FEDER & 3.500 & 5.000 \\
\hline Préstamos BEI & 24.580 & 42.000 \\
\hline TOTAL & 55.158 & 124.367 \\
\hline
\end{tabular}

(*) en millones de euros; (a) 1996-1999; (b) 1993-1999.

FUENTE: CCE, 2002, 5; PLANCO, 2003, 94-105. 
Mapa 3. Proyectos Prioritarios de la RTE-T en la UE ampliada

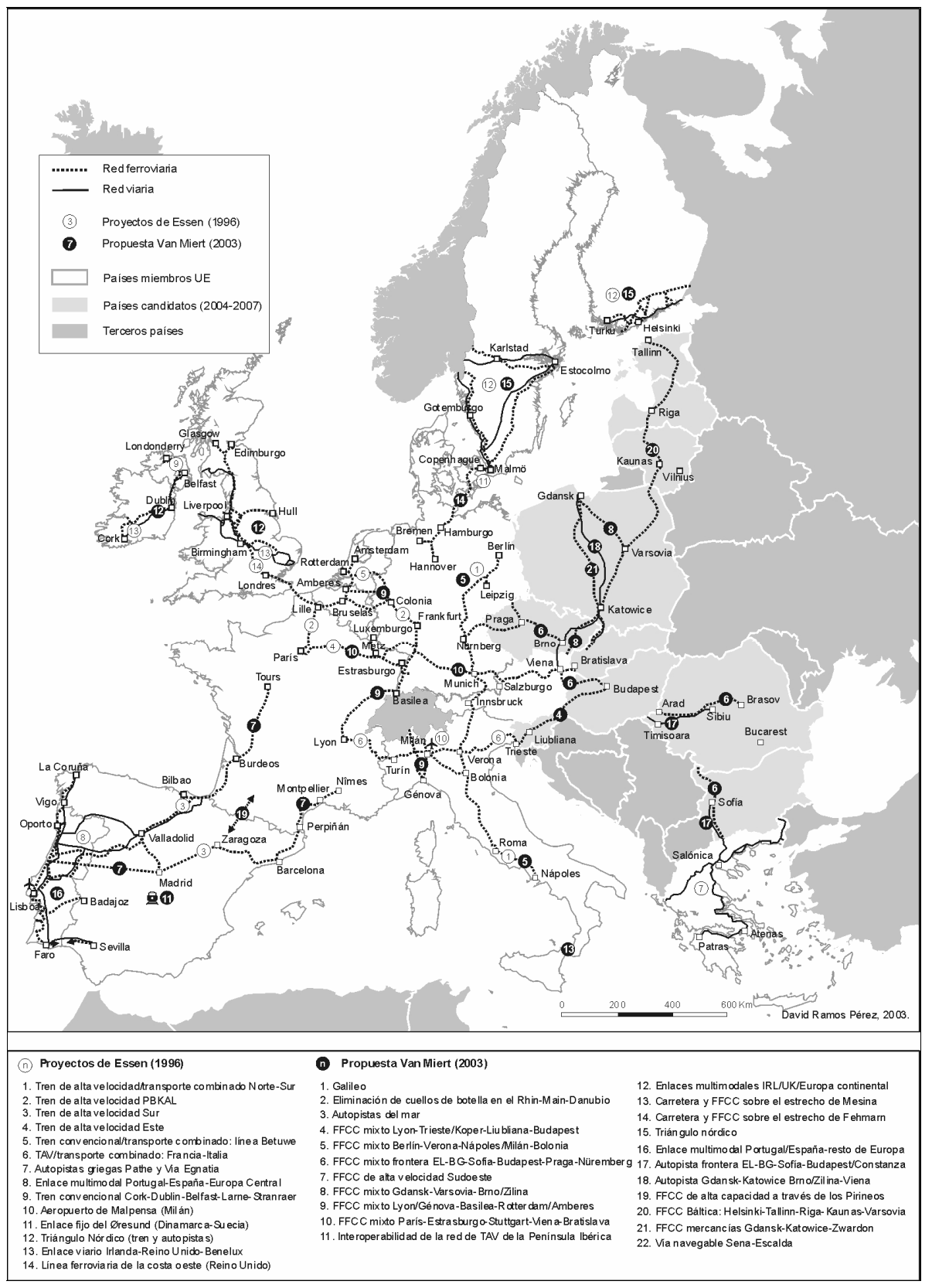

FUENTE: CCE, 2003. Elaboración Propia. 
A estas ayudas directas podemos añadir los préstamos del Banco Europeo de Inversiones (BEI), que si bien han sido considerables hasta 1999, esperan superar los 40.000 millones hasta el 2006. En cambio, el énfasis puesto desde 1996 en las asociaciones público privadas (APP) para hacer frente a la inversión de los proyectos prioritarios, ha dado escasos frutos, sobre todo por la resistencia de la iniciativa privada para involucrarse en unas actuaciones en las que están implicados distintos países, con diferentes escenarios administrativos, legislativos y políticos (SICHELSCHMIDT, 1999, 174). A pesar de todo, la preocupación mostrada reiteradamente por la Comisión respecto a la posibilidad de incumplir los plazos previstos debido a la falta de inversión, parece hasta cierto punto infundada. Algunos estudios recientes refuerzan esta idea, ya que la consultora PLANCO estima que tres cuartas partes de la inversión necesaria para completar en 2010 la RTE-T ya está consignada en los distintos presupuestos (PLANCO, 2003, 109-110), y el Informe Van Miert afirma que ocho de los proyectos prioritarios estarán finalizados en 2010 (tres de ellos ya han concluido), y amplias secciones de los seis restantes también se ultimarán antes de 2010 (CCE, 2003).

\section{La ampliación de la RTE-T hacia el Este: los Corredores Paneuro- peos y el proyecto TINA.}

El interés de la Unión Europea en construir una red de transporte más allá del territorio conformado por sus Estados miembros quedó puesta de manifiesto mucho antes de la aprobación en 1996 de las orientaciones de la RTE-T. Así, en 1991, de forma paralela al lanzamiento del programa de RTE-T, el Parlamento Europeo y la Comisión organizaron en Praga la Primera Conferencia Paneuropea de Transporte, con la finalidad de iniciar una estrecha cooperación a escala continental en materia de política de transportes e inversión en infraestructuras. La tercera de estas reuniones, celebrada en Helsinki en 1997, supuso un notable avance, al proponer la Comisión una estructura de redes de transporte para el conjunto del continente, donde la RTE-T ya definida en el seno de la UE y su extensión a los países candidatos tuviera un papel prominente ${ }^{3}$. Surgieron así 10 Corredores Paneuropeos de Transporte (MAPA 4), considerados ejes fundamentales para avanzar en la mejora de la articulación física entre la Europa Oriental y Occidental. De esta manera se pretenden facilitar los desplazamientos de larga distancia y eliminar los estrangulamientos existentes, especialmente en las zonas fronterizas.

\footnotetext{
${ }^{3}$ Esta propuesta se recoge en la comunicación presentada en 1997 por la Comisión La conexión de la red de infraestructuras de transporte de la Unión con los países vecinos - hacia una política paneuropea de cooperación en las redes de transporte (CCE, 1997a).
} 
Mapa 4. Corredores Paneuropeos de Transporte

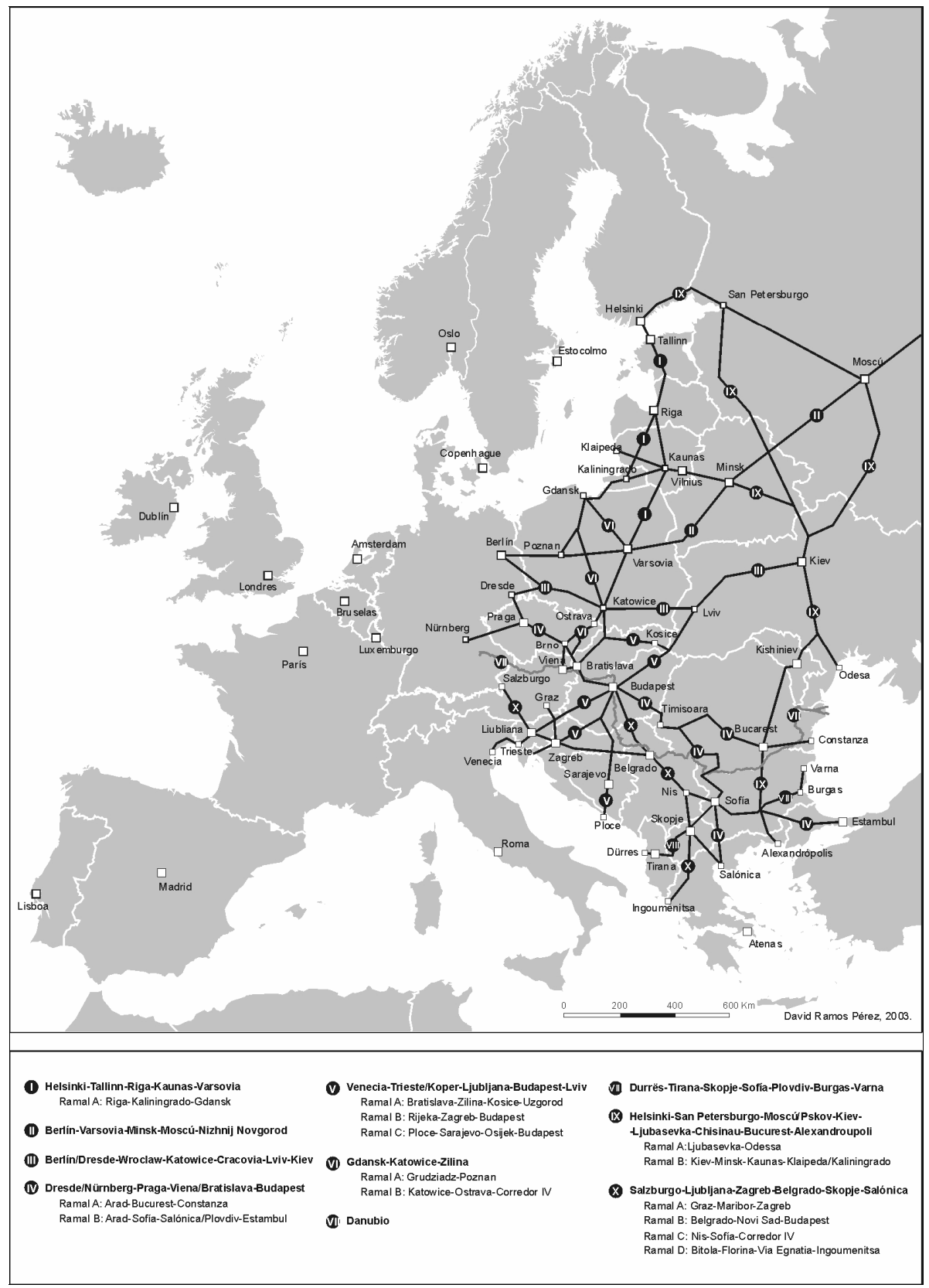

FUENTE: CEMAT, 2000; TINA, 2002. Elaboración Propia. 
Estos Corredores Paneuropeos constituyen la estructura principal de la denominada red TINA ${ }^{4}$, definida entre 1995 y 1999 por un grupo de trabajo que evaluó las necesidades en infraestructura de transporte de los países candidatos a la adhesión $^{5}$, de cara a una futura ampliación de la RTE-T (TINA, 1999, 12). La red TINA, que a esa estructura principal añade una serie enlaces complementarios (MAPAS 1 y 2), quedó conformada por $18.700 \mathrm{~km}$ de carreteras (el 17,3\% de la red principal de los países candidatos), $20.924 \mathrm{~km}$ de ferrocarriles (el $31,5 \%), 20.000 \mathrm{~km}$ de vías navegables, 40 aeropuertos, 20 puertos marítimos y 58 puertos fluviales. Se cumplía así con algunos de los objetivos y grandes líneas de actuación recogidos en las orientaciones de la RTE-T, en los que se insta a la cooperación con países terceros afectados por el programa, con la finalidad de aumentar la interoperabilidad y homogeneidad de las redes. No en vano, se prevé que la ampliación puede generar un crecimiento explosivo de los intercambios de bienes y personas, como ya se viene constatando desde 1990. Entre esa fecha y el año 1998 los países candidatos duplicaron el volumen de las exportaciones hacia la UE15 y multiplicaron por 5 las importaciones, lo que ha saturado algunos ejes este-oeste y creado puntos de fuerte congestión en la frontera entre Alemania y Polonia (CCE, 2001b, 103).

La red TINA definida también debe cumplir, según el informe final de 1999, con los objetivos recogidos en las orientaciones sobre la RTE-T, haciéndose especial referencia a aquellos contenidos en el artículo 154 del Tratado. De esta manera, la red se convierte en herramienta para el buen funcionamiento del mercado interior y la cohesión económica y social en el este del continente, equiparándose su papel con el asignado a la RTE-T. Por otro lado, las actuaciones seleccionadas se consideran básicas para el buen desarrollo de los Corredores Paneuropeos, puesto que los países de Europa Central y Oriental afectados por la red TINA constituyen en territorio de tránsito natural para las conexiones entre la actual UE y las nuevas repúblicas surgidas de la desaparición de la URSS y Yugoslavia.

En el momento actual se estima que el coste de las obras propuestas superará los 90.000 millones de euros durante el periodo 1999-2015, aunque a largo plazo se especula con un programa más ambicioso, que incluya nuevas conexiones y se aproxime a los 260.000 millones. A diferencia de lo que ocurre con la RTE-T, la carretera acapara una inversión mayor, que ronda el $50 \%$ de lo presupuestado (CUADRO 3), lo que se justifica en parte por el mal estado y menor

\footnotetext{
${ }^{4}$ El acrónimo TINA deriva del nombre original inglés del programa, Transport Infrastructure Needs Assessment.

${ }^{5}$ En un primer momento participaron Bulgaria, República Checa, Eslovaquia, Eslovenia, Estonia, Hungría, Letonia, Lituania, Polonia, Rumania y Chipre, sumándose posteriormente Malta.
} 
desarrollo de la red viaria en estos países, donde las autopistas son todavía muy escasas $\left(0,3 \mathrm{~km}\right.$ cada $100 \mathrm{~km}^{2}$, mientras la media de la UE15 alcanza los 1,6 $\mathrm{km}$ ). De todas formas, análisis recientes muestran que por el momento los países candidatos tienen planificado invertir hasta el 2015 menos de 50.000 millones de euros (PLANCO, 2003, 62-64). Al igual que ocurre con la RTE-T, son los presupuestos nacionales los que deben soportar la mayor parte de la inversión, y la situación económica de los futuros miembros de la UE todavía no les permite gastos excesivos en infraestructura. Las ayudas provenientes de los fondos comunitarios son muy escasas para un programa tan ambicioso: los 905 millones de euros invertidos por el PHARE entre 1991 y 1998, y los 3.500 previstos en el nuevo Instrumento de Política Estructural de Preadhesión (ISPA), entre 2000 y 2006, se antojan cantidades excesivamente reducidas para poder cumplir con los objetivos previstos.

Cuadro 3. Composición y coste de la red TINA (1999-2015)

\begin{tabular}{lrrr}
\hline \multicolumn{1}{c}{ Modos } & Longitud o n & Coste $\left(^{*}\right)$ & $\%$ \\
\hline Red viaria & $18.683 \mathrm{~km}$ & 44.304 & 48,4 \\
Red ferroviaria & $20.924 \mathrm{~km}$ & 37.119 & 40,5 \\
Navegación interior & $4.052 \mathrm{~km}$ & 1.493 & 1,6 \\
Aeropuertos & 40 & 4.416 & 4,8 \\
Puertos marítimos & 20 & 2.944 & 3,2 \\
Puertos fluviales & 58 & 306 & 0,3 \\
Terminales & 86 & 1.012 & 1,1 \\
\hline TOTAL & & 91.594 & 100,0 \\
\hline
\end{tabular}

(*) Estimaciones en millones de euros.

FUENTE: TINA, 1999, 31.

En última instancia, parece que comienzan a sentarse los cimientos de una Red Paneuropea de Transporte, compuesta por la RTE-T, la red TINA, los tramos de los Corredores Paneuropeos de Transporte no incluidos en esta última, y las denominadas Zonas Paneuropeas de Transporte, también definidas en la Conferencia de Helsinki de 1997 y consideradas como regiones específicas, abiertas a fachadas marítimas extensas, donde el concepto de corredor no satisface sus necesidades de infraestructura ${ }^{6}$. A estas redes y zonas suelen añadirse los futuros enlaces euroasiáticos, especialmente el Corredor de Transporte Europa-Cáucaso-Asia, conocido como TRACECA (TINA, 2002, 7). Para el Conse-

\footnotetext{
${ }^{6}$ Se trata de cuatro zonas, Mar de Barents / Euro-Ártica, Cuenca del Mar Negro, Cuenca Mediterránea y Mar Adriático / Mar Jónico. Salvo en el caso de la primera, donde el Consejo de los Estados del Mar de Barents promueve diversas iniciativas de cooperación, los avances registrados son escasos, limitándose a definir las interconexiones con la RTE-T o la red TINA.
} 
jo de Europa esta red debería ser el punto de partida de una verdadera política paneuropea de transporte, que responda a los retos de la creciente congestión y presión sobre el medio ambiente (CEMAT, 2000, 11). Sin embargo, por el momento, no parece que se apueste decididamente por ir más allá de una simple definición grandes corredores de transporte que faciliten los desplazamientos de larga distancia.

\section{LA RED TRANSEUROPEA DE TRANSPORTE O LA REFORMU- LACIÓN DEL BINOMIO TRANSPORTE-DESARROLLO.}

\section{El transporte como motor del desarrollo regional.}

Una vez conocido con cierto detalle el proceso de gestación de la RTE-T, la magnitud del proyecto, las distintas fórmulas de financiación del mismo y su extensión hacia el este como consecuencia del proceso de ampliación de la UE, conviene ahora evaluar hasta qué punto la RTE-T ha cumplido con los objetivos que justificaron la puesta en marcha del programa. Ello implica adentrarse en la controversia sobre la vinculación existente entre transporte y desarrollo económico, pues no olvidemos que en última instancia la filosofía que sustenta la RTE-T se fundamenta en ese binomio.

La relación de causalidad establecida entre transporte y desarrollo se ha mantenido prácticamente estable desde la Revolución Industrial, acompañando en función de la época a la construcción del ferrocarril, de las carreteras y autopistas y, más recientemente, del tren de alta velocidad (OFFNER, 1993, 234). De hecho, esta relación continúa proporcionando a la Economía del Transporte su marco de análisis básico, ya que parte de la consideración del transporte como una forma de producción, es decir, de generación de riqueza (ESTEVAN Y SANZ, 1996, 36). En este sentido, los análisis más simples de la economía clásica afirman que una mejora en las infraestructuras generará inferiores costes de transporte y un incremento de los procesos competitivos. Al mismo tiempo, la mayor facilidad de acceso a las materias básicas por parte de las empresas locales incrementará su productividad y competitividad frente a otras que se sitúen en regiones con un menor nivel de accesibilidad (VICKERMAN ET AL., 1999, 1-2). En los documentos preparatorios de la RTE-T, la Comisión se identificaba claramente con este razonamiento, al afirmar que las redes "pueden reducir la distancia de ciertas regiones a los actuales centros económicos y favorecer el desarrollo de actividades económicas" (CCE, 1990, 16). Y en esta idea se ha apoyado desde entonces la defensa de la contribución de la RTE-T a la cohesión económica y social, tal y como recogen los objetivos de las orientaciones comunitarias de 1996. 
Limitándonos a entender la cohesión económica y social como la reducción de diferencias entre los niveles de desarrollo de las diversas regiones de la UE, tal y como se desprende del artículo 158 del Tratado, la vinculación que realiza la Comisión entre la RTE-T y la cohesión parece, cuando menos, demasiado simple. ¿Un menor tiempo de acceso desde la periferia al centro de la Unión va a impulsar de forma decisiva el desarrollo de determinadas regiones desfavorecidas? Es verdad que la mejora de los enlaces entre las regiones centrales y las periféricas facilitará a las empresas de las últimas la comercialización de sus productos, pero de forma paralela, los productores de las regiones centrales pueden invadir los mercados periféricos previamente protegidos por su relativa lejanía (VICKERMAN ET AL., 1999, 2), al tiempo que la necesidad de contar con oficinas filiales se reduce drásticamente.

Continuando con la misma línea argumental, si las infraestructuras de transporte fueran capaces de inducir tales cambios en la periferia, las disparidades regionales en el seno de la UE ya habrían mostrado una cierta tendencia a atenuarse, puesto que buena parte de la ayuda proveniente de los fondos estructurales se destina a la construcción de las mismas en las regiones objetivo 1, que habitualmente combinan un menor desarrollo con la lejanía respecto a los espacios más dinámicos de la UE. La inversión en estas infraestructuras supuso el 18,7\% del gasto total del FEDER en el periodo 1989-1993, descendió hasta el $15,7 \%$ en el periodo $1994-1999$ y está previsto que ronde el $19 \%$ entre 2000 y 2006. Sin embargo, los estudios realizados hasta el momento muestran que las disparidades regionales no disminuyen, y como mucho tienden a estabilizarse. Aunque el Segundo Informe sobre la Cohesión económica y social se muestre relativamente optimista (CCE, 2001d, 4-6), la realidad es que entre 1988 y 1998, el PIB per cápita, expresado en unidades de poder adquisitivo y considerando la media de la UE igual a 100 , del $25 \%$ de las regiones más prósperas pasó de 134,1 a 137,1 mientras el del 25\% de las regiones más pobres aumentaba del 66,6 al 68,3 .

Igualmente significativos son los resultados que arrojan las proyecciones a largo plazo sobre la capacidad la RTE-T para mitigar las diferencias regionales. Así, el modelo SASI desarrollado por la Universidad de Dortmund muestra que, si en el año 2016 estuvieran concluidos todos los proyectos de la red viaria y ferroviaria incluidos en la RTE-T, se observaría una distribución ligeramente menos polarizada de la accesibilidad y el PIB per cápita entre las regiones, pero sería tan leve que se mostraría incapaz de revertir la tendencia general hacia la concentración económica en la UE (FÜRST ET AL., 2000, 59). Para los autores, ello demuestra que factores como el envejecimiento de la población, la movilidad de la mano de obra y la productividad de la misma, son mucho más relevantes de cara al desarrollo regional y tiene un impacto más destacado en la co- 
hesión que la simple inversión en infraestructura de transporte. De esta manera, se pone de manifiesto la complejidad que existe para analizar el verdadero papel de los transportes en la transformación del espacio social y económico y los efectos de las infraestructuras en el desarrollo económico (PLASSARD, 1994: $38)$.

\section{Vertebrar nodos, desarticular territorios.}

Aún sabiendo que la reducción de los tiempos de desplazamiento no es un factor determinante del desarrollo, deberíamos cuestionarnos hasta qué punto la RTE-T beneficia realmente a la periferia en términos de accesibilidad. Aunque es verdad que la RTE-T, al ser el resultado de las negociaciones entre los distintos Estados miembros, cubre la práctica totalidad del territorio de la UE, existen razones para dudar que las obras en marcha incidan realmente en las regiones periféricas. Una de estas razones es la existencia de 14 proyectos prioritarios, que consumen más del $25 \%$ de la inversión total del programa, orientados fundamentalmente a la construcción de ferrocarriles de alta velocidad y excesivamente concentrados en las áreas más dinámicas del territorio comunitario. Una visualización espacial de estos proyectos (MAPA 3) muestra que no afectan al centro y sur de Italia, ni al oeste de Francia y suroeste de España, ni tampoco al norte de Escandinavia (ROBERT, 2001, 68). Por tanto, gran parte de la inversión beneficia a las principales aglomeraciones urbanas de la banana azul o dorsal europea del desarrollo, donde no sólo se concentra la riqueza, sino que ya disfrutan de una elevada centralidad.

Considerando únicamente los efectos generados por la red transeuropea de ferrocarril de alta velocidad, VICKERMAN ET AL. $(1999,10-12)$ han constatado la ampliación en las diferencias de accesibilidad entre las regiones centrales y periféricas de la UE entre 1993 y 2010. Aunque ciudades de la periferia, como Estocolmo, Madrid o Lisboa, incrementan su accesibilidad con la nueva infraestructura, son las aglomeraciones localizadas en la banana azul las que obtienen mayores beneficios, ya que con la red finalizada experimentan un notable aumento de la población que puede ser alcanzada desde ellas en viajes de ida y vuelta en el mismo día. No en vano, el énfasis dado a la conexión entre el centro y la periferia, hace que crezcan aún más las facilidades para acceder desde el centro a todo el territorio de la UE, mientras la interrelación entre las distintas periferias apenas mejora (CCE, 1996: 77).

Pero, ¿Estocolmo, Madrid y Lisboa forman parte de la periferia? La distinción entre centro y periferia no es simple, ya que no se encuentra únicamente relacionada con una determinada localización geográfica. Así, dentro de espacios geográficamente periféricos es posible distinguir nodos urbanos dinámicos y prósperos, cada vez más estrechamente vinculados con la dorsal europea, y que 
escapan a la consideración de periferia. En este sentido, las ciudades antes mencionadas, y otras como Helsinki, Atenas, Copenhague o Barcelona se convierten también en centros del territorio europeo, a pesar de encontrarse distantes y geográficamente desconectadas de la banana azul. Las comunicaciones fluidas que se establecen entre ellas y el tradicional espacio central de la UE, facilitadas fundamentalmente por el transporte aéreo y las telecomunicaciones, permiten avanzar en la definición de un territorio central en red (VELTZ, 1999, 64), donde se aprecian crecientes interdependencias entre los nodos que lo componen. Y el tren de alta velocidad no hace sino potenciar las relaciones entre ellos, porque no olvidemos que se trata de un modo de transporte espacialmente muy selectivo, cuya finalidad es enlazar grandes aglomeraciones urbanas obviando el territorio intermedio, considerado mero soporte de la infraestructura y sin apenas conexiones a la red (efecto túnel).

Estas constataciones llevan a que nos preguntemos por el destino de la inversión del Fondo de Cohesión en el ámbito del transporte, una de las vías de financiación de la RTE-T que teóricamente debiera prestar especial atención a las regiones periféricas. Las ayudas de este Fondo se destinan a los países más desfavorecidos de la UE, con renta per cápita inferior al $90 \%$ de la media comunitaria. Pero como el objetivo del Fondo es nacional y no regional, los proyectos subvencionados no tienen por qué afectar únicamente a las regiones más pobres, lo que ha posibilitado una distribución poco progresiva de la ayuda (Roger TYM \& PARTNERS, 1996, 64). Así, Lisboa e Vale do Tejo, Cataluña, la Comunidad Valenciana, Madrid y Attiki se encuentran entre las regiones que más fondos reciben en términos absolutos, siendo las más desarrolladas de sus respectivos países. Por ello no debe extrañarnos que cerca del $84 \%$ del gasto en infraestructura realizado por España entre 1999 y 2001 con cargo al Fondo de Cohesión se haya destinado al tren de alta velocidad Madrid-Barcelona, línea que conecta las principales ciudades del país y que reporta escaso beneficio al territorio intermedio: sólo existen paradas en Guadalajara, Calatayud, Zaragoza y Lérida.

Se corre por tanto el riesgo de conectar las principales ciudades de las regiones geográficamente periféricas con la dorsal europea mediante redes de transporte de excelencia, como el tren de alta velocidad o las autopistas, pensado que así se consigue un aumento generalizado de la accesibilidad, no sólo de las regiones donde se insertan, sino incluso de sus respectivos países. El efecto resultante es el contrario, porque esta estrategia termina fomentando las disparidades entre las ciudades privilegiadas y sus respectivos hinterlands, debido al menor desarrollo e inferior calidad de las redes secundarias, lo que priva de mejoras en la accesibilidad a aquellos que habitan en las verdaderas periferias. 
Los primeros trabajos sobre la ordenación del territorio a escala comunitaria ya avisaban que el desarrollo de la RTE-T podía ser un factor de desequilibrio, dada la amplitud de las mallas que formaría esa red principal de autopistas y ferrocarril de alta velocidad, lo que podría crear "espacios vacíos, incluso desiertos, entre los puntos y corredores de concentración, si no se mejora simultáneamente la accesibilidad de estas zonas mediante el desarrollo de las redes secundarias" (CCE, 1994, 64). El diseño de la RTE-T, y muy especialmente de sus proyectos prioritarios, muestra claramente que se da preferencia a la alta velocidad, a la larga distancia y al tráfico internacional, decisiones que favorecen a las zonas más prósperas de la UE. En este sentido, sería necesario fortalecer otras redes, mejorando las infraestructuras de transporte locales y regionales del continente, con la idea de impulsar ciclos de producción, distribución y consumo a escala regional (T\&E ET AL., 2003, 15), que no sólo pueden potenciar el desarrollo de las áreas periféricas sino que contribuyen de forma efectiva a una estrategia de movilidad sostenible.

Todo ello lleva a tomar conciencia de la poca adaptación de la RTE-T en su definición actual a las necesidades reales de las regiones periféricas. Es muy discutible que una red ferroviaria de alta velocidad, fundamentalmente orientada al tráfico de pasajeros, vaya a reportar grandes ventajas al usuario, que encuentra en el transporte aéreo el modo más óptimo de conexión entre la periferia y el centro. Muy al contrario, los efectos ocasionados por la práctica eliminación del ferrocarril convencional en esos corredores, el cierre de estaciones y el aumento de los precios tendrán una influencia claramente regresiva desde el punto de vista de la equidad social y territorial. Es por ello que la periferia necesita de fórmulas alternativas en el ámbito del transporte, adaptadas a las necesidades específicas de cada caso, y buscando siempre la mejora de la articulación a escala regional y la conexión de las periferias entre sí. En el primer aspecto, la inversión en redes secundarias, tanto viarias como ferroviarias, y el desarrollo de la intermodalidad, serán vitales para lo cohesión interna de los territorios. En el segundo, la aviación regional debe desempeñar un papel trascendental, evitando la concentración de los beneficios derivados de la liberalización del transporte aéreo en un número reducido de nodos y posibilitando enlaces directos entre aeropuertos secundarios.

\section{3. ¿Repitiendo los errores en Europa Central y Oriental...?}

La reflexión crítica realizada a lo largo de este apartado nos ha permitido vislumbrar algunos de los riesgos asociados a una inversión masiva en infraestructura de transporte que, carente de una rigurosa evaluación de sus posibles efectos, puede terminar generando escenarios totalmente contradictorios con los objetivos inicialmente planteados. Lamentablemente, la extensión de la RTE-T hacia el este del continente, por medio de la red TINA, no supone un replan- 
teamiento estratégico del programa, ya que nuevamente se apuesta por grandes corredores de tránsito que faciliten el transporte a larga distancia en el menor tiempo posible.

La transición de una economía planificada al libre mercado, con la consiguiente reestructuración productiva, ha marcado profundamente el devenir de los países de Europa Central y Oriental durante la última década. Es innegable que de estos cambios derivan notables repercusiones en cuanto a la orientación de la demanda de transporte, debido al fuerte declive de las relaciones comerciales con la antigua Unión Soviética y el auge de los intercambios con la UE, sobre todo desde 1992. Al mismo tiempo, es un hecho que buena parte de su infraestructura de transporte se encuentra en un lamentable estado de conservación, debido a la ausencia de inversiones durante las últimas décadas. Sin embargo, ello no justifica la repetición de un modelo marcado por una estrategia de crecimiento económico a corto plazo, que olvida las necesidades de desarrollo equilibrado y sostenible a largo plazo.

El incremento de las disparidades regionales, tanto desde el punto de vista de los ingresos como del empleo, ha sido uno de los resultados negativos del proceso de transición hacia la economía de mercado. En este sentido, se constata la consolidación del modelo de islas de crecimiento formulado a principios de los noventa, que pronosticaba la aparición de un número reducido de regiones con un fuerte crecimiento económico, cada vez más distanciadas del resto de territorios, afectados por una grave crisis estructural (CCE, 1996b, 110-115). Las regiones urbanas donde se asientan las distintas capitales nacionales constituyen el principal exponente de estas islas de crecimiento, en las que el nivel de prosperidad es muy superior a la media nacional, y en los casos de Praga, Bratislava, Budapest y Liubliana cercano a la media comunitaria.

En este contexto, es previsible que la mayor parte del gasto en infraestructura de transporte se destine a las regiones más prósperas y a la mejora de las conexiones entre ellas, siendo mucho menores los fondos dirigidos a las áreas menos favorecidas. La limitada capacidad de financiación pública acentuará este proceso, ya que se recurrirá a las concesiones a compañías privadas para la materialización de los proyectos, y como resulta lógico, estas empresas preferirán los centros de crecimiento económico antes que las periferias con la finalidad de acelerar la rentabilización de la inversión. Todo ello no hará sino favorecer la polarización de las actividades económicas en un número restringido de espacios, acrecentando los desequilibrios territoriales ya existentes, con una clara tendencia a marginar las regiones orientales y los espacios intersticiales entre los principales corredores de transporte, convertidos en nuevas periferias de la Unión Europea. 
Por otro lado, existe un alto riesgo de exportar a los países de Europa Central y Oriental los patrones de movilidad de la UE, en los que dominan los modos de transporte más ineficientes. El crecimiento en los niveles de motorización de los países candidatos (de 146 a 223 vehículos cada 1.000 habitantes entre 1990 y 1998), el rápido declinar de la cuota de mercado del transporte público, el aumento de la distancia recorrida por persona y día en las ciudades, el cierre de casi $4.000 \mathrm{~km}$ de vías férreas durante la década de los noventa, junto con la construcción de 700 kilómetros de autopistas en ese mismo periodo, son claros indicios de los cambios que comienzan a operarse (EEA, 2002, 31-32; T\&E, $2002,16)$. Convertir el automóvil y las autopistas en símbolos del desarrollo constituye un grave error, sobre todo cuando desde las instituciones comunitarias se trata de impulsar cambios que reorienten la demanda hacia otros modos.

Una de las herencias de los regímenes comunistas es la densa red ferroviaria de los países candidatos y el elevado uso del transporte público en las ciudades. Sobre esta base debería ser posible construir un modelo de transporte que, inserto en una economía de libre mercado, sirva de referencia para el cambio que debe llevarse a cabo en los países de Europa Occidental. Es evidente la necesidad de invertir en determinadas autopistas y mejorar la red viaria en su conjunto, pero ello no debe hacerse a costa del modo ferroviario, porque se estarían repitiendo paso a paso los errores ya cometidos en otros lugares en el pasado.

Sin embargo, los datos sobre la inversión ejecutada y la prevista a corto y medio plazo no nos permiten ser muy optimistas. Si entre 1993 y 1999 el porcentaje del gasto destinado a ambos modos ha sido similar (45\%), en el periodo 2000-2003 la carretera ya acaparó el 60\% de la inversión y el ferrocarril el $30,5 \%$, situación que se agravará entre 2004 y 2015 donde el dinero comprometido hasta el momento se destina en un $80 \%$ a la red viaria, dejando apenas un $16 \%$ para el tren. Del reciente análisis realizado por PLANCO $(2003,64)$ se desprende, en primer lugar, la más que probable incapacidad de los futuros Estados miembros para afrontar la inversión presupuestada para la red TINA entre 1999 y 2015, superior a los 90.000 millones de euros. Y, en segundo lugar, tal y como hemos comprobado, la clara preferencia dada a la carretera ante un escenario de reducción del gasto global en infraestructura de transporte.

Al igual que la RTE-T, el diseño de la red TINA no se adapta a las necesidades reales de las regiones periféricas y menos desarrolladas de los países candidatos, posibilitando además el descenso de la cuota de mercado del ferrocarril en el transporte de mercancías, al facilitar el trasvase hacia la carretera. En cierta medida, las disfunciones que se aprecian en la red TINA derivan de un proceso de planificación poco transparente y carente de una evaluación estratégica que permita una aproximación al impacto real de las infraestructuras previstas. Además, no olvidemos que la red TINA se trata de un proyecto puesto en mar- 
cha en el seno de la UE, y que fundamentalmente responde a las preocupaciones comerciales de los Estados miembros. Aunque es verdad que los países candidatos estuvieron presentes durante su gestación, su capacidad de maniobra para proponer alternativas era reducida, sobre todo teniendo en cuenta que las ayudas económicas de la UE en materia de transporte iban a favorecer estos corredores (T\&E, 2002, 8). Por ello, las posibilidades de un cambio de rumbo parecen, por el momento, muy limitadas.

\section{CONCLUSIONES.}

Transcurridos más de quince años desde el inicio oficial del programa de RTE-T, los resultados finales derivados de su materialización están aún lejos de conocerse. Desde un punto de vista teórico, la iniciativa de la RTE-T debe contribuir al cumplimiento de muchos de los objetivos de la Unión Europea, desde el refuerzo de la cohesión económica y social hasta la mejora de la seguridad, pasando por la protección ambiental y el incremento de las relaciones con los países vecinos (Ross, 1998, 182). Sin embargo, a nuestro juicio, no existen fundamentos científicos que evidencien la estrecha relación entre la RTE-T y ese amplio abanico de objetivos. Es más, tal y como se ha expuesto a lo largo de las páginas precedentes, parece que la propia implantación de la red, especialmente en lo que respecta al ferrocarril de alta velocidad, puede generar escenarios claramente negativos para la cohesión y el equilibrio territorial en el seno del continente.

Aunque desde hace décadas se acepta que las infraestructuras de transporte constituyen una condición necesaria pero en modo alguna suficiente para el desarrollo, la RTE-T parece sustentarse básicamente en esa relación de causalidad, ampliada y mejorada, al convertir al transporte en uno de los motores de la cohesión económica y social. Por tanto, las regiones periféricas deberían ser las grandes beneficiadas del programa, frente a unas regiones centrales que ya cuentan con unas óptimas dotaciones, aunque congestionadas por el excesivo tráfico que soportan. Los análisis realizados muestran que se ha preferido mitigar la congestión de las regiones prósperas, antes que cumplir con el objetivo de reducir el aislamiento de la periferia, debido al temor de una pérdida de competitividad del conjunto de la UE. Únicamente los principales centros urbanos de las regiones periféricas obtienen ciertos beneficios, lo que acentúa las diferencias entre ellos y sus hinterlands, propiciando el incremento de los desequilibrios.

Por otro lado, es un mito común considerar el transporte como una fuerza exclusivamente descentralizadora, que favorece la difusión de las actividades a lo largo del espacio. Muy al contrario, en la mayoría de las ocasiones el transporte conduce a una fuerte centralización, y las nuevas infraestructuras suelen limitar- 
se a reforzar los patrones de intercambio y relación previamente existentes (Rodrigue et al., 2002). Generalmente se olvida que la mejora de la accesibilidad no es unívoca, ya que las regiones centrales también se benefician de ella, lo que posibilita la ampliación de sus áreas de influencia y una mayor polarización territorial.

La UE aspira a que la RTE-T sustente el modelo de desarrollo policéntrico propuesto en la Estrategia Territorial Europea, evitando así la excesiva concentración de la riqueza y la población en la banana azul y su entorno inmediato. Pero es poco probable que el actual programa de RTE-T impulse el equilibrio territorial del continente, siendo previsible que acentúe las disparidades y desequilibrios entre las distintas regiones. Ello deriva en parte de la escasa transparencia y reducida participación de los agentes regionales en el proceso de gestación, lo que dio lugar a un diseño de las distintas redes que responde sobre todo a los intereses de los principales grupos industriales de la UE. Se refuerza así una organización económica donde priman las relaciones y el intercambio a larga distancia en un tiempo cada vez menor, olvidándose las necesidades de las comunidades periféricas y aisladas, progresivamente excluidas de ese patrón de crecimiento.

En este contexto cobra cada vez mayor sentido el preguntarse si el desarrollo económico y equilibrado de los territorios de Europa no debiera sustentarse en un gran número de pequeños proyectos a escala regional más que en grandes inversiones alejadas de las problemáticas cotidianas de muchos espacios (RoBERT, 2001, 67). Para ello debe darse mayor énfasis a las redes secundarias, adaptadas a las condiciones locales específicas, y favorables a la revitalización de los ciclos de producción y consumo de ámbito regional. Algunos autores van aún más lejos, señalando que si se quiere convertir a la RTE-T en un verdadero instrumento para la cohesión entre las regiones de Europa, es necesario un cambio significativo en la orientación de las inversiones, de tal forma que se favorezcan los enlaces en el interior de las regiones periféricas y entre éstas, "no en adición, sino a costa de la inversión que se lleva a cabo en el centro de Europa" (VICKERMAN ET AL, 1999,13).

Pero este cambio parece lejano. El proceso de revisión de la RTE-T iniciado en 2001 por la Comisión (CCE, 2001c) no modifica en lo sustancial las orientaciones de 1996, centrándose las discusiones en la identificación de nuevos proyectos prioritarios. Aprovechando la coyuntura de la inminente ampliación de la UE, el Informe Van Miert ha propuesto la renovación de la lista de proyectos prioritarios, añadiendo nuevas intervenciones en los países candidatos e incluyendo sustanciales extensiones en algunos proyectos de Essen todavía inconclusos (MAPA 3). Se consolida de esta manera un modelo de RTE-T muy selectivo, que apuesta por grandes proyectos cuya capacidad de vertebración del te- 
rritorio europeo es más que discutible. Más grave aún es la total opacidad en la elaboración de un informe que condiciona la toma de decisiones políticas y compromete una fuerte inversión económica. Que el antiguo comisario Van Miert no haya comparecido ante el Parlamento Europeo durante el proceso de redacción del mismo, a pesar de la insistencia de algunos europarlamentarios, confirma el escaso interés por abrir un debate siquiera institucional sobre la RTE-T.

Finalmente, el intento de trasladar miméticamente a los países candidatos el modelo de organización del transporte dominante en Europa Occidental, evidencia que aún se concede escasa importancia a las externalidades negativas derivadas de una movilidad claramente insostenible a largo plazo. Los países de Europa Central y Occidental constituían, en su mayoría, un entorno adecuado para avanzar decididamente en un nuevo modelo de movilidad y en una nueva concepción de las infraestructuras de transporte, que sirvieran de impulso para el desarrollo endógeno de las distintas regiones y permitieran, en última instancia, la construcción de un territorio mucho más equilibrado. Casi con toda seguridad la oportunidad se ha perdido.

\section{BIBLIOGRAFÍA}

Asamblea De Las Regiones De EuRopa (ARE) (1996): Regions and Territories in Europe. The region's View of the Territorial Effects of European Policies. Estrasburgo, Asamblea de las Regiones de Europa.

COMISIÓN DE LAS COMUNIDADES EUROPEAS (CCE) (1989): Hacia unas redes transeuropeas: objetivos y aplicaciones posibles (COM (89) 643). Luxemburgo, Oficina de Publicaciones Oficiales de las Comunidades Europeas.

COMISIÓN DE LAS COMUNIDADES EUROPEAS (CCE) (1990): Hacia unas redes transeuropeas: programa de actuación comunitario (COM (90) 585 final). Luxemburgo, Oficina de Publicaciones Oficiales de las Comunidades Europeas.

COMISIÓN DE LAS COMUNIDADES EUROPEAS (CCE) (1992): El curso futuro de la política común de transportes - Un enfoque global para la elaboración de un marco comunitario de movilidad sostenible (COM (92) 494). Luxemburgo, Oficina de Publicaciones Oficiales de las Comunidades Europeas.

COMISIÓN DE LAS COMUNIDADES EUROPEAS (CCE) (1994): Europa 2000+: Cooperación para la ordenación del territorio europeo. Luxemburgo, Oficina de Publicaciones Oficiales de las Comunidades Europeas.

COMISIÓN DE LAS COMUNIDADES EUROPEAS (CCE) (1996a): Primer Informe sobre la Cohesión económica y social. Luxemburgo, Oficina de Publicaciones Oficiales de las Comunidades Europeas.

COMISIÓN DE LAS COMUNIDADES EUROPEAS (CCE) (1996b): The impact of the development of the countries of Central and Eastern Europe on the 
Community territory. Luxemburgo, Oficina de Publicaciones Oficiales de las Comunidades Europeas.

COMISIÓN DE LAS COMUNIDADES EUROPEAS (CCE) (1997a): La conexión de la red de infraestructuras de transporte de la Unión con los países vecinos hacia una politica paneuropea de cooperación en las redes de transporte (COM (97) 172). Luxemburgo, Oficina de Publicaciones Oficiales de las Comunidades Europeas.

COMISIÓN DE LAS COMUNIDADES EUROPEAS (CCE) (1997b): Perspectiva Europea de Ordenación del Territorio - Primer borrador oficial. Luxemburgo, Oficina de Publicaciones Oficiales de las Comunidades Europeas.

COMISIÓN DE LAS COMUNIDADES EUROPEAS (CCE) (1999): Estrategia Territorial Europea. Hacia un desarrollo equilibrado y sostenible del territorio de la UE. Luxemburgo, Oficina de Publicaciones Oficiales de las Comunidades Europeas.

COMISIÓN De LAS COMUNIDADES EUROPEAS (CCE) (2001a): Desarrollo sostenible en Europa para un mundo mejor: Estrategia de la Unión Europea para un desarrollo sostenible (COM(2001) 264 final). Luxemburgo, Oficina de Publicaciones Oficiales de las Comunidades Europeas.

COMISIÓN DE LAS COMUNIDADES EUROPEAS (CCE) (2001b): La política europea de transportes de cara al 2010: la hora de la verdad (COM(2001) 370). Luxemburgo, Oficina de Publicaciones Oficiales de las Comunidades Europeas.

COMisión De Las COMUnidades EuROPeAS (CCE) (2001c): Propuesta de Decisión del Parlamento Europeo y del Consejo por la que se modifica la Decisión $n^{\circ}$ 1692/96/CE del Parlamento Europeo y del Consejo sobre las orientaciones comunitarias para el desarrollo de la red transeuropea de transporte. Luxemburgo, Oficina de Publicaciones Oficiales de las Comunidades Europeas.

COMISIÓN DE LAS COMUNIDADES EUROPEAS (CCE) (2001d): Segundo Informe sobre la Cohesión económica y social. Luxemburgo, Oficina de Publicaciones Oficiales de las Comunidades Europeas.

COMISIÓN DE LAS COMUNIDADES EUROPEAS (CCE) (2002): Trans-European Transport Network: TEN-T priority projects. Luxemburgo, Oficina de $\mathrm{Pu}-$ blicaciones Oficiales de las Comunidades Europeas.

COMISIÓN DE LAS COMUNIDADES EUROPEAS (CCE) (2003): High level group on the Trans-European Transport Network - Report. Luxemburgo, Oficina de Publicaciones Oficiales de las Comunidades Europeas.

CONFERENCIA EUROPEA DE MinisTROS RESPONSABLES DE ORDENACIÓN DEL TERRITORIO (CEMAT) (2000): Principios Directores para el Desarrollo Territorial Sostenible del Continente Europeo. Estrasburgo, Consejo de Europa. 
DiARIO OfICIAL DE LAS COMUNIDADES EuROPEAS (DOCE) (1995): Reglamento (CE) $n^{\circ} 2236 / 95$ del Consejo, de 18 de septiembre de 1995, por el que se determinan las normas generales para la concesión de ayudas financieras comunitarias en el ámbito de las redes transeuropeas (L228 de 23-91995). Luxemburgo, Oficina de Publicaciones Oficiales de las Comunidades Europeas.

DiARIO OFICIAL DE LAS COMUNIDADES EuROPEAS (DOCE) (1996): Decisión 1692/96/CE del Parlamento Europeo y del Consejo, de 23 de julio de 1996, sobre las orientaciones comunitarias para el desarrollo de la red transeuropea de transporte (L228 de 9-9-1996). Luxemburgo, Oficina de Publicaciones Oficiales de las Comunidades Europeas.

ESTEVAN, A. y SANZ, A. (1996): Hacia la reconversión ecológica del transporte en España. Madrid, Los Libros de la Catarata.

EUROPEAN ENVIRONMENTAL AGENCY (EEA) (2002): Paving the way for EU enlargement. Indicators of transport and environment integration - TERM 2002. Luxemburgo, Oficina de Publicaciones Oficiales de las Comunidades Europeas.

FÜrst, F., SChÜrmann, C., SPIEKERMAnN, K. y WEgEnER, M. (2000): The SASI (Socio-Economic and Spatial Impacts of Transport Infrastructure Investments and Transport System Improvements) Model: Demonstration Examples. Dortmund, IRPUD.

OFFNER, J. M. (1993): «Les effets structurants du transport: mythe politique, mystification scientifique», L'Espace Géographique, 3, 233-242.

PIODI, F. (1997): The Financing of Trans-European Transport Networks (Transport Series - Working Document E-4). Luxemburgo, Parlamento Europeo.

PLANCO (2003): TEN - Invest. Essen, PLANCO Consulting GmbH.

PLASSARD, F. (1994): Le transport a grande vitesse et le développment régional. Comunicación presentada en la Mesa Redonda de la Conferencia Europea de Ministros de Transporte: Politiques régionales, réseaux de transport et communications, París.

RICHARDSON, T. (1997): «The Trans-European Transport Network: Environmental policy integration in the European Union», European Urban and Regional Studies, 4, 4, 333-346.

ROBERT J. (COORD) (2001): Spatial impacts of Community Policies and costs of non-co-ordination. Luxemburgo, Dirección General de Política Regional de la Comisión Europea.

RoDRIGUE, J. P. (Ed.) (2002): Transport Geography on the Web. Hofstra University, Department of Economics \& Geography. [Disponible en http://people.hofstra.edu/geotrans]. 
ROGER TYM \& PARTNERS (1996): The regional impact of Community policies (Regional Policy Series - Working Paper W-16). Luxemburgo, Parlamento Europeo.

Ross, J. F. L. (1998): Linking Europe. Transport Policies and Politics in the European Union. Westport y Londres, Praeger.

SCHMIDT, M. y GIORGI, L. (2001): «Successes, Failures and Prospects for the Common Transport Policy», Innovation, 14, 4, 293-313.

SICHELSCHMIDT, H. (1999): «The EU programme "trans-European networks" a critical assessment», Transport Policy, 6, 169-181.

T\&E (2002): Be smart, do it better! - A guide to sustainable transport in accession countries (T\&E 02/6). Bruselas, European Federation for Transport and Environment.

T\&E, WWF, BIRDLIFE INTERNATIONAL, CEE BANKWATCH NETWORK y FOEE (2003): Trans-European Transport Networks - Options for a sustainable future (T\&E 03/2). Bruselas, European Federation for Transport and Environment.

TINA SECRETARIAT (1999): TINA - Transport Infrastructure needs assessment - Final Report. Viena, Dirección General de Transportes y Dirección General de Asuntos Exteriores de la Comisión Europea.

TINA SECRETARIAT (2002): Status of the Pan-European Transport Corridors and Areas - Developments and Activities in 2000 and 2001. Viena, Dirección General de Energía y Transportes de la Comisión Europea.

Veltz, P. (1999): Mundialización, ciudades y territorios. Barcelona, Ariel.

VICKERMAN, R., SPIEKERMANN, K. y WEGENER, M. (1999): «Accessibility and Economic Development in Europe», Regional Studies, 33, 1, 1-15.

RESUMEN: La Red Transeuropea de Transporte (RTE-T) constituye una de las iniciativas más ambiciosas de la Unión Europea (UE), tanto por la magnitud de los objetivos perseguidos como por la escala de actuación y la elevada inversión económica que supondrá su materialización efectiva. Sin embargo, el programa de RTE-T no deja de ser un producto refinado derivado de la ya clásica relación de causalidad establecida entre transporte y desarrollo económico, binomio que permite justificar políticamente los grandes gastos en infraestructura. Además, el diseño actual de la RTE-T puede acentuar más que mitigar las disparidades regionales, siendo por tanto discutible su contribución al objetivo de la cohesión económica y social. La próxima ampliación de la UE debería abrir el debate sobre el futuro de la RTE-T, impulsando un nuevo modelo de red que no traslade al este del continente los impactos negativos conocidos y posibilite a largo plazo un cierto reequilibrio territorial de Europa.

PALABRAS CLAVE: Red de transporte, cohesión, equilibrio territorial, Europa. 
ABSTRACT: The Net Transeuropea of Transport (RTE-T) one of the most ambitious initiatives in the European Union constitutes (UE), so much for the magnitude of the objectives pursued as for the performance scale and the high economic investment that it will suppose their effective materialization. However, the program of RTE-T doesn't stop to already be a derived refined product of the classic relationship of established causation between transport and economic development, binomial that allows to justify the big expenses politically in infrastructure. Also, the current design of the RTE-T can accentuate more than to mitigate the regional disparities, being therefore debatable its contribution to the objective of the economic and social cohesion. The next amplification of the UE should open the debate on the future of the RTE-T, impelling a new net model that doesn't transfer to the east of the continent the well-known negative impacts and facilitate long term a certain territorial reequilibrio of Europe.

KEY WORDS: Transport network, cohesion, balanced spatial structure, Europe.

$\boldsymbol{R} \boldsymbol{E} \boldsymbol{S U} \boldsymbol{M E} \boldsymbol{E}:$ Le Réseau transeuropéen de transport (RTE-T) est l'une des initiatives les plus ambitieuses de l'Union Européenne (UE), aussi bien par l'ampleur des objectifs poursuivis que par le fort niveau d'engagement et d'investissement financier nécessaires à sa future mise en place. Cependant, le programme RTE-T reste un produit raffiné conséquence du rapport de causalité, désormais classique, entre transport et développement économique, un binôme qui permet de justifier politiquement les dépenses élevées en infrastructure. En outre, l'actuelle conception du RTE-T pourrait aggraver, au lieu de mitiger, les disparités régionales, en mettant en cause sa contribution à l'objectif de cohésion économique et sociale. Le prochain élargissement de l'UE devrait lancer le débat sur l'avenir du RTE-T et développer un nouveau modèle de réseau qui ne déplace pas à l'est du continent les effets négatifs connus, permettant à long terme un certain rééquilibre territorial en Europe.

MOTS-CLÉS: Réseau de transport, cohésion, équilibre du territoire, Europe.

RESUMO: A Rede Transeuropeia de Transporte (RTE-T) constitui uma das iniciativas mais ambiciosas da União Europeia (UE), tanto pela magnitude dos objectivos perseguidos quanto pela escala de actuação e o elevado investimento económico que vai supor a sua materialização efectiva. Porém, o programa da RTE-T não deixa de ser um produto requintado derivado da já clássica relação de causalidade estabelecida entre transporte e desenvolvimento económico, binómio que permite justificar politicamente as grandes despesas em infra-estrutura. Além disso, o design actual da RTE-T pode acentuar mais do que mitigar as disparidades regionais, sendo portanto discutível a sua contribuição ao objectivo da coesão económica e social. A próxima ampliação da UE deveria abrir o debate acerca do futuro da RTE-T, impulsionando um novo modelo de rede que não transfira para leste do continente os impactos negativos conhecidos e possibilite a longo prazo um certo reequilíbrio territorial da Europa.

$\boldsymbol{P A L A V R A S ~ C H A V E}$ : Rede de transporte, coesão, equilíbrio territorial, Europa. 